\title{
Encryption and Compression Digital Video Files using Artificial Neural Networks and Genetic Algorithm
}

\author{
Hasan M.A. Anwer \\ Department of Software Engineering \\ College of Computer Science and Mathematics \\ University of Mosul \\ hasanmaher@uomosul.edu.iq \\ DOI: $\underline{10.33899 / \text { edusj.1970.163335 }}$ \\ Received \\ Accepted \\ 20/ 06/ 2018 \\ $02 / 10 / 2018$
}

\begin{abstract}
The research and studies of encryption and compression of digital files to the confidentiality and ease of dealing with networks and the Internet, by reducing the size of files transmitted through multimedia, and also used to increase the confidentiality of information transmitted.

The purpose of the research is to encrypt and compress colored digital video files using artificial neural networks and genetic algorithm, The digital video file is entered into Audio Video Interleave (AVI) and cut into a set of frames, Each frame is then analyzed into the three color segments (red, green and blue) And an audio input from the Waveform Audio File Format (WAV) type (which represents the encryption key), the encoded frame was then inserted into the compression algorithm, A hybrid compression method was adopted which is considered as one of the solutions that reduces the size of the required data on the one hand and gives it a secret on the other, Where it was segmentation and clustering the encoded frames into a cluster of clusters (The optical pixels), which have been introduced into the artificial neural network (back propagation network), The genetic algorithm has been used to calculate the weight values of each cell of the network. The decryption and decompression is then performed, and by using a set of testing and comparison tools that showed that the results were consistent with the research requirements, and Matlab R2017b was used to implement research algorithms.
\end{abstract}

Keywords: Encryption, compression, clustering, digital video, BPN, GA. 


\section{تثفير وكبس ملفات الفيديو الرقمي باستخدام الثبكات العصبية الاصطناعية والخوارزمية}

$$
\text { الجينية }
$$

$$
\begin{aligned}
& \text { حسن ماهر أحمد أنور } \\
& \text { قسم هندسة البرمجيات } \\
& \text { كلية علوم الحاسوب والرياضيات } \\
& \text { جامعة الموصل }
\end{aligned}
$$

hasanmaher@uomosul.edu.iq

DOI: $\underline{10.33899 / \text { edusj.1970.163335 }}$

$$
\text { القبول }
$$

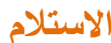

\section{الخلاصة}

تهدف البحوث والدراسات الخاصة بتشفير وكبس الملفات الرقمية إلى سرية وسهولة التعامل مع شبكات الاتصالات والانترنيت، وذلك من خلال تقليل حجم الملفات المنقولة عبر الوسائط المتعددة، وكنلك تستعمل لزيادة سرية المعلومات المنقولة. يهدف البحث إلى تشفير وكبس ملفات الفيديو الرقمي الملون باستعمال الثبكات

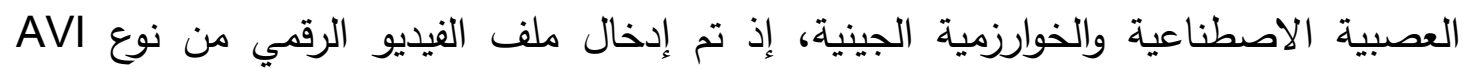
وتقطيعه إلى مجموعة من الأطر ثم تحليل كل إطار إلى الثرائح اللونية الثلاث (الأحمر

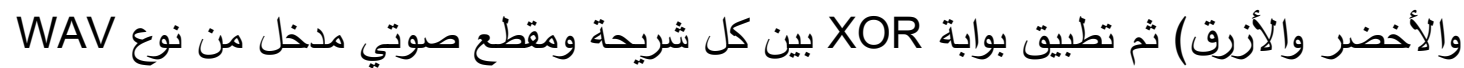

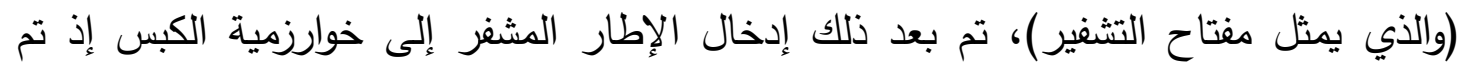

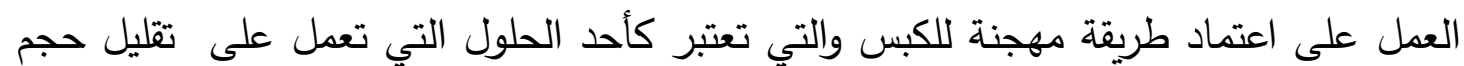
البيانات المطلوبة من جهة وإعطائها سرية من جهة أخرى، إذ تم تقسيم وعنقدة الأطر المشفرة

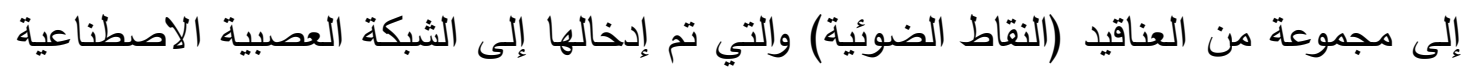
(شبكة انتشار الخطأ خلفيا)، وقد تم الاعتماد على الخوارزمية الجينية في حساب قيم الأوزان لكل

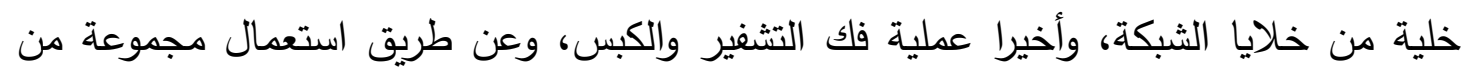

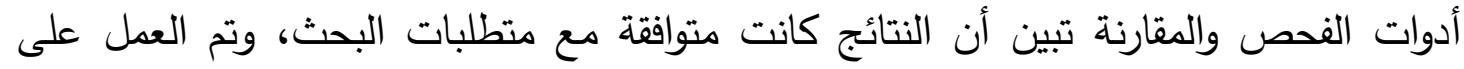
استعمال لغة Matlab R2017b لتنفيذ خوارزميات البحث. الكلمات المفتاحية: تشفير، كبس، العنقدة، الفيديو الرقمي، شبكة الانتشار العكسي، الخوارزمية 
:Introduction المقدمة-1

في السنوات الأخيرة ازدادت ضخامة المعلومات الصورية المخزونة التي تعالج رقمياً في مختلف

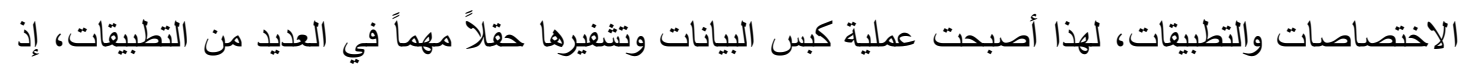
أن معظم أنظمة التشغيل الحديثة تعتمد على الرسومات أو الصور ، ولكون بيانات الفيديو الرقمي تحتل مساحة كبيرة في الذاكرة أدى ذلك إلى الاهتمام الكبير في إيجاد تقنيات لكبس البيانات الفيديوية أو الصورية[1].

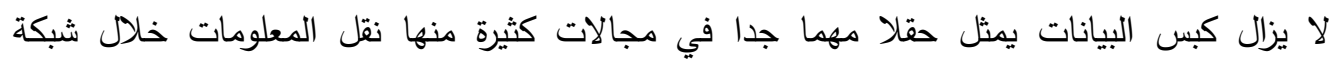
الاتصالات الرقمية فضلا عن أهميته في تقليل مساحات الخزن في الذاكرة المستعملة لخزن هذه البيانات وأهميته

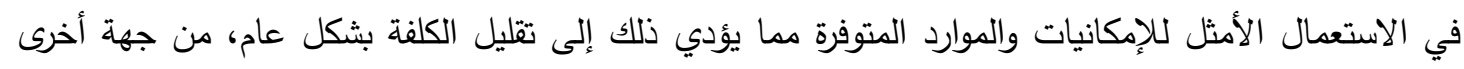
يؤدي إلى تقليل وقت نقل الملف المستعمل وتقليل حزمة الإرسال (Bandwidth).

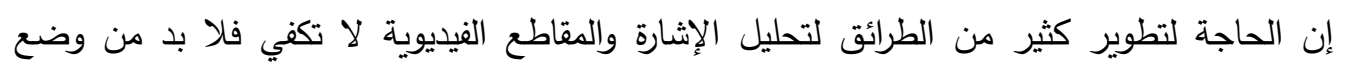
وتطوير طرائق لكبسها، إذ إن هناك عدة تقنيات لكبس البيانات ويعتمد اختيار التقنية على نوع البيانات المراد

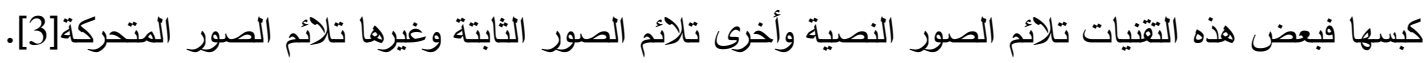

2-2 الاراسات السابقة Previous studies:

مع تطور أجهزة الحاسوب الرقمية التي دخلت تطبيقاتها كافة مجالات الحياة وأصبح التطور في مجال الحاسوب وتطبيقاته المختلفة التي تتراوح من التطبيقات الصناعية إلى الأبحاث الفضائية احد أهم مقاييس تطور الدول في العصر الحديث فقد قدم العديد من الباحثين دراسات متتوعة في هذا المجال، ففي عام 2017 اقترح الباحثان Choudhary و بحثا تم من خلاله تشفير صورة رقمية بطريقة مبنية على الخوارزمية الجينية

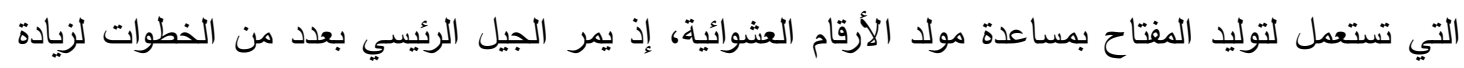

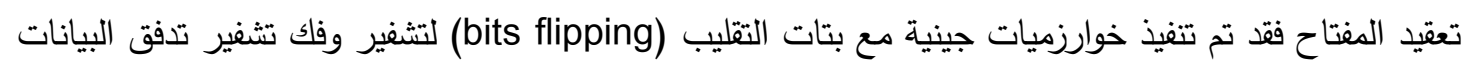

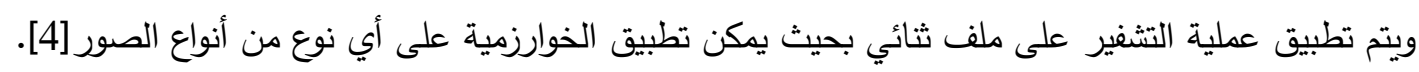

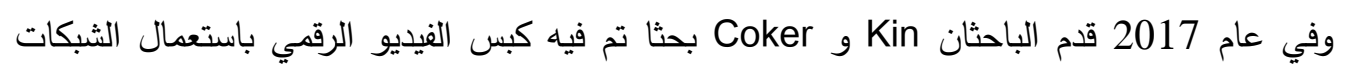
العصبية الاصطناعية إذ عمد الباحثان على تقديم نظامًا للتشفير التلقائي يتكون من شبكات عصبية فئية تلاففية متكررة ثنائية الاتجاه (Recurrent Convolutional Neural Networks) لكبس الفيديو واستتتج الباحثان من خلال النتائج الى أن شبكات RCNN يمكن أن تتعلم من المعلومات الزمنية الموجودة في إطارات ثابتة متتالية ولكنها تفشل في تحقيق معدلات كبس وسرعات متطورة جدًا بسبب التعقيدات الحسابية [3]. وفي عام 2018 قدم الباحثان Zhang و Wu بحثا اقترحا فيه طريقة لتقليل نسبة فقد البيانات في الصور الرقمية المكبوسة عبر دمج معيار الدقة في تصميم الثبكة العصبية بحيث لا يمكن إسقاط أو تشويه أي هياكل صغيرة ومميزة من الصورة الأصلية علاوة على ذلك تم تصميم الثبكة العصبية المضادة للقطع الأثرية للعمل على مجموعة من معدلات بتات الكبس بدلاً من مجموعة ثابتة كما في الماضي واظهرت النتائج التجريبية أن الطريقة المقترحة يمكنها استعادة تفاصيل الصورة الدقيقة التي يتم تدميرها أو تفتيتها بخوارزميات أخرى [5]. 


\section{3- المخطط العام للبحث General Research Plan:}

إن الحاجة لتطوير كثير من الطرائق لتحليل الإشارات والصور المتتالية لا تكفي ولكن يجب أن يتم

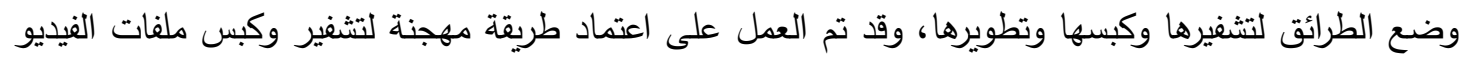
الرقمي والتي تعتبر كأحد الحلول التي تعمل على تقليل حجم البيانات المطلوبة من جهة وإعطائها سرية من جهة

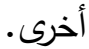

تم في هذا البحث إدخال ملف الفيديو وتقطيعه إلى مجموعة من الأطر (وهي بمثابة صور رقمية متتالية

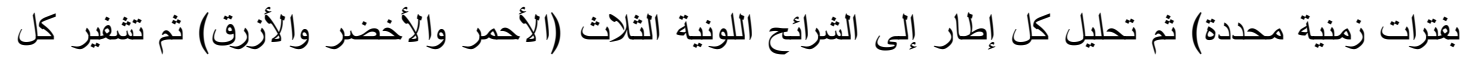
شريحة ليتم إدخالها إلى الشبكة العصبية (شبكة انتشار الخطأ خلفيا)، وقد تم الاعتماد على الخوارزمية الجينية في حساب قيمة الأوزان لكل خلية من خلايا الشبكة، وكما في المخطط الانسيابي (1).

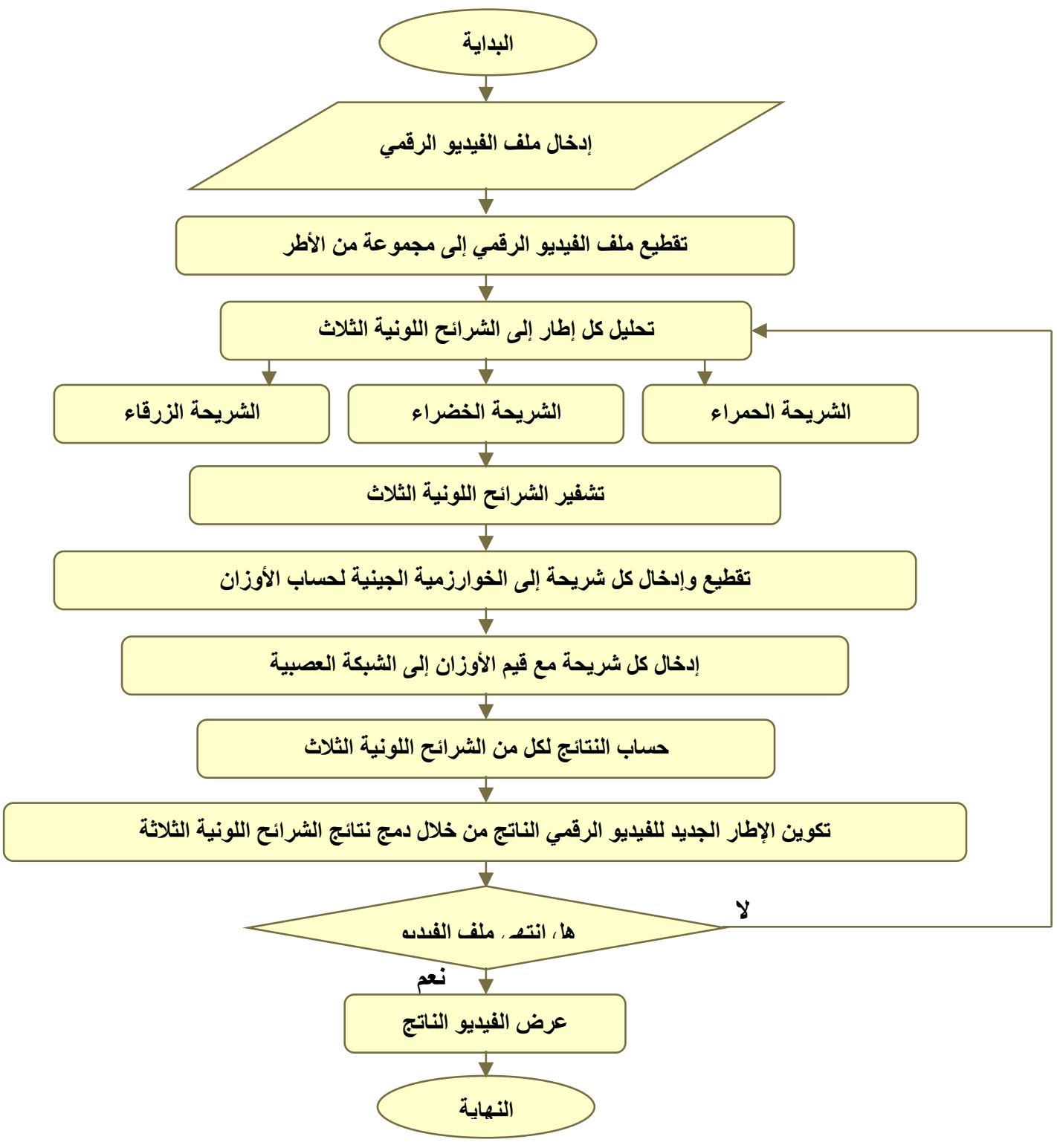

المخطط الانسيابي (1): المخطط العام للبحث 
تم العمل على إدخال ملفات الفيديو الرقمي ذات الامتداد (AVI)، وذلك لأنها تحتوي على صيغة

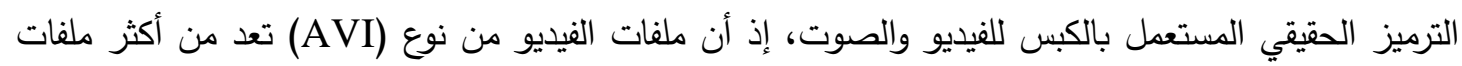

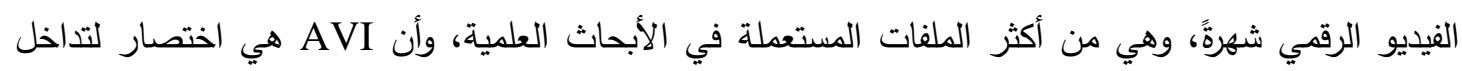

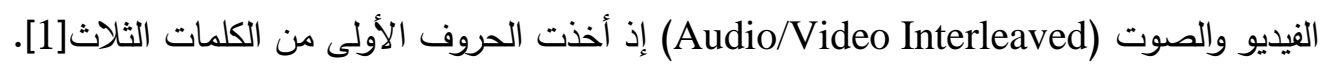

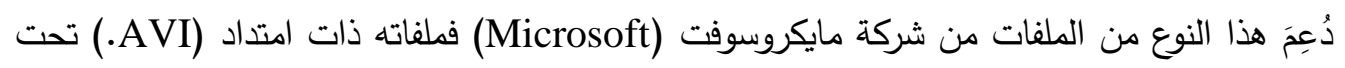

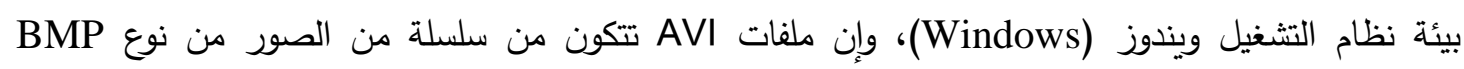

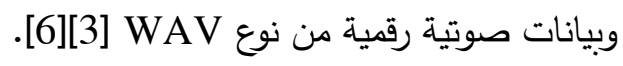

\section{4- التشفير والعنقدة Encryption and clustering:}

علم التشفير (Cryptography) هو العلم الذي يعنى بالطرق التي تعمل على حماية المعلومات ونقلها

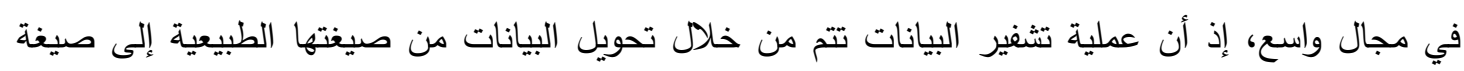
أخرى غامضة غير قابلة للفهم عبر مجموعة من العمليات والخطوات بهدف حماية البية فيانات أو إرسالها لأطراف

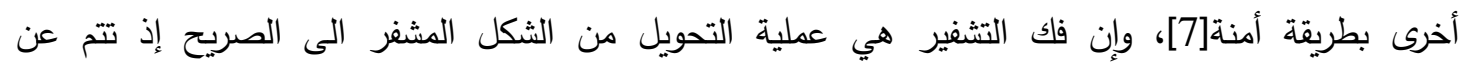
طريق مفتاح التثفير ، وتقسم خوارزميات التشفير حسب طريقة العمل على اجزاء ورموز البيانات إلى نوعين[8]:

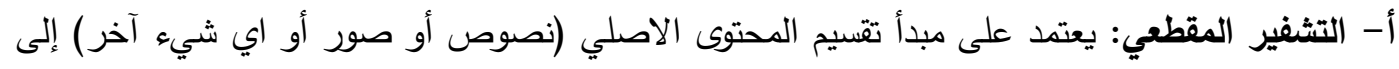

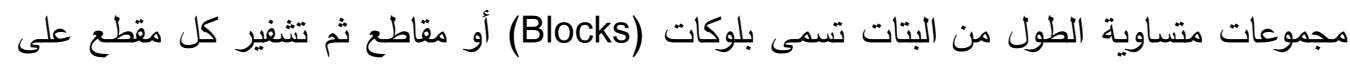

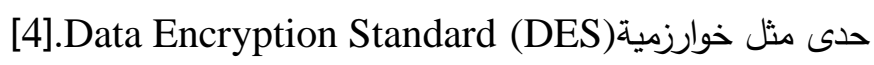
ب- التثفير المتصل: ويعتمد على مبدأ تشفير البيانات المتصل أو جدول البيانات بشكل مستمر إذ يتم توليد مفتاح مستمر يتم دمجه مع البيانات الأصلية بخوارزمية تشفير ذات مفتاح متماثل وغالبا يتم ذلك لكئك بعملية XOR المنطقية وهو ما اعتمد عليه البحث.

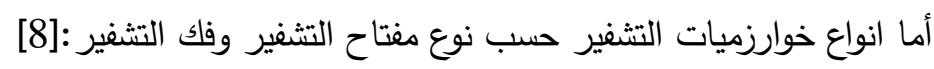
أ- التشفير المتناظر (symmetric systems): إذا يستعمل نفس المفتاح في التشفير وفلك التشفير حيث يقوم نظام التشفير المتماثل باستعمال نفس المفتاح في التشفير وفك التشفير ومن مزايا التشفير

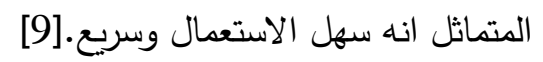
ب- التشفير غير المتناظر (asymmetric systems): يتم استعمال مفتاح للتشفير واخر لفك التشفير وباستعمال هذين الزوجين من المفاتيح إحداهما عام (public) والآخر خاص (private) يستطيع

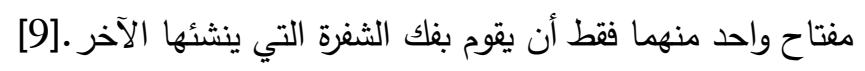
أما خوارزمية التثفير فهي مجموعة من العمليات التي تهدف إلى تحويل بيانات أطر الفيديو المدخلة إلى اطر فيديو مشفرة، فبعد الحصول على الشرائح الثلاث الملونة يتم تحويل كل شريحة إلى مصفوفة أحادية

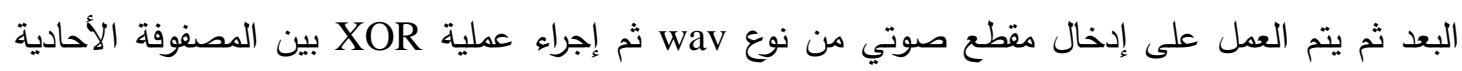
والمقطع الصوتي، وكما موضح في الثكل (1)، والمخطط الانسيابي (2)، لتتتج مصفوفة أحادية مشفرة والتي لتي ستطبق عليها خطوات الكبس. 
حسن ماهر أحمد أنور

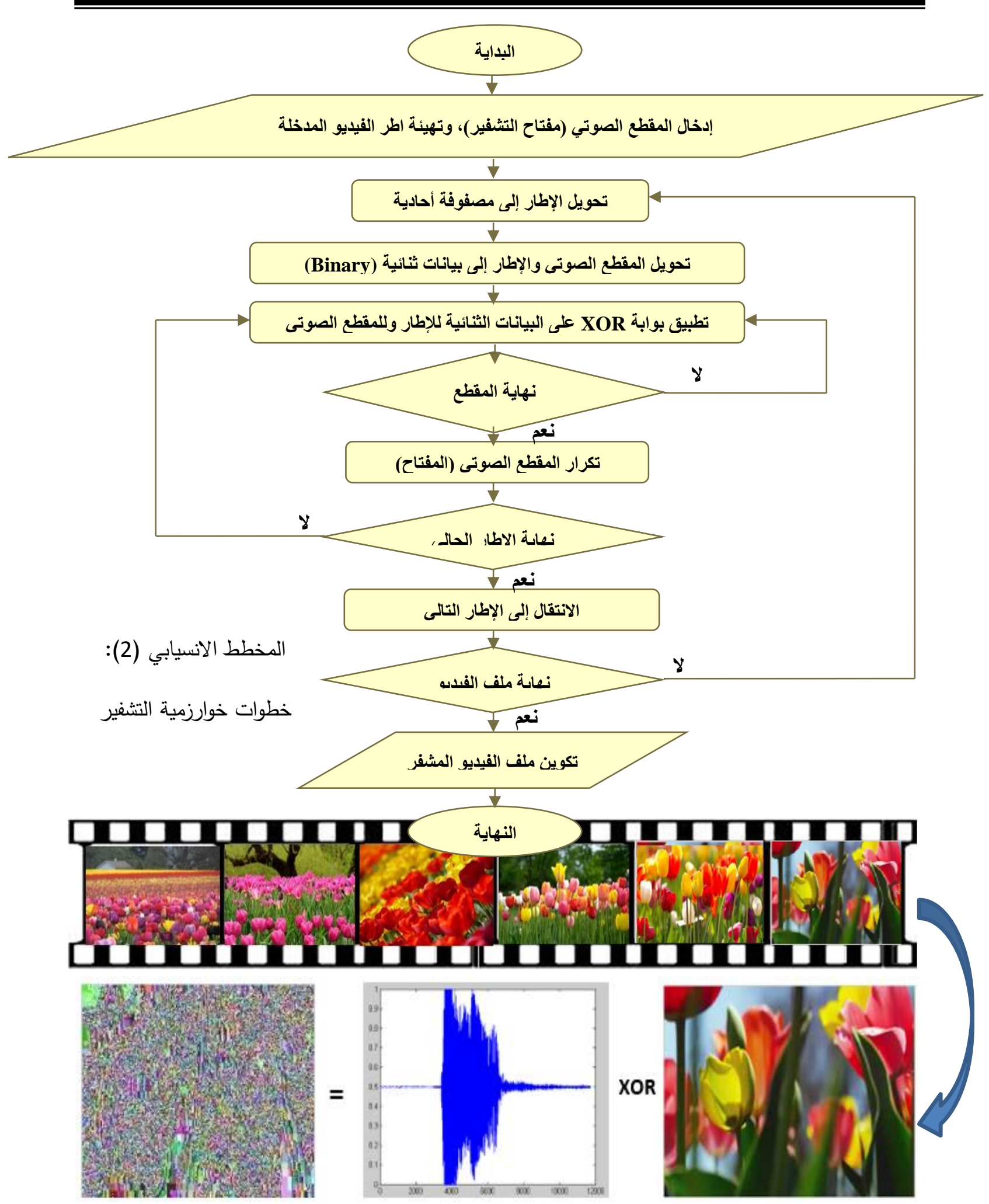

الشكل (1): تشفير الإطار الأول من ملف الفيديو

بعد تثفير اطر الفيديو المدخل تم تقطيع كل شريحة لونية إلى مجموعة من النقاط الضوئية (pixels) فمثلا (2*2) إذ يتم في كل مرة إدخال أربعة نقاط ضوئية إلى الشبكة العصبية الاصطناعية. 
إن الفكرة العامة من مسألة العنقدة تتلخص بتجزئة مجموعة من البيانات المعطاة إلى مجاميع (عناقيد) إذ أن نقاط البيانات في العنقود الواحد تكون متشابهة مع بعضها البعض أكثر من تلك النقاط في العناقيد الأخرى[10]، إذ أن العنقدة هي عملية تقسيم البيانات إلى مجاميع اعتمادا على بعض المقائيس المتثابهة لهذه

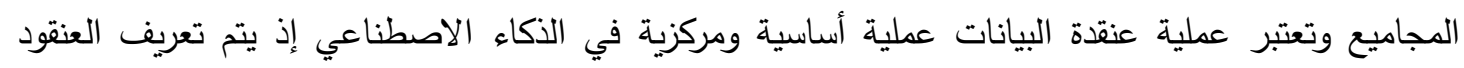
بواسطة مركز العنقود والطريقة الأكثر شيوعا لإيجاد التشابه بين البيانات ومراكز العناقيد هي الاعتماد على [11].(Euclidean distance) المسافة الاقليدية إن عملية العنقدة هي مفهوم جديد في مجال الكبس يعمل على تجميع الأثياء مثل النقاط في الصورة

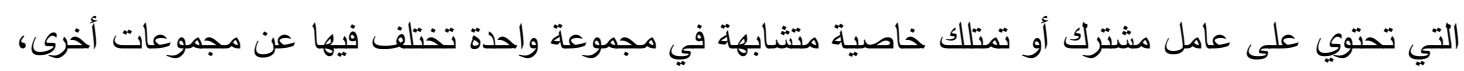
قد يكون هذا التشابه بين العناصر في المجموعة الواحدة (العنقود) هو البعد بين كل من هذه العناصر عن نقطة فئنة

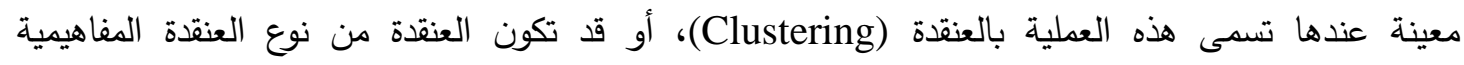
(Conceptual Clustering)

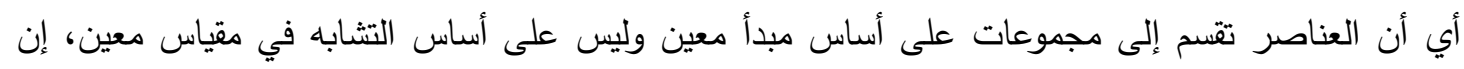

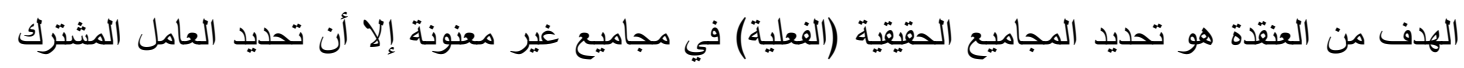

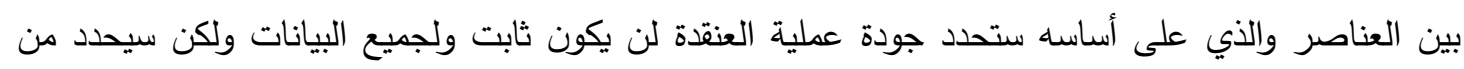

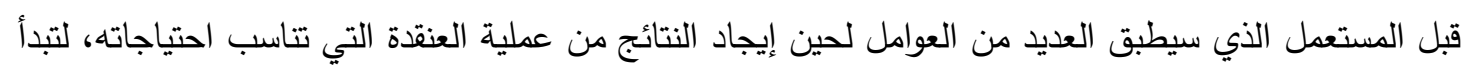
بعدها عملية الكبس.

يعرف الكبس بأنه تقنية تستعمل لتقليل حجم ملف البيانات الرقمية لغرض التقليل من المساحة الخزينة

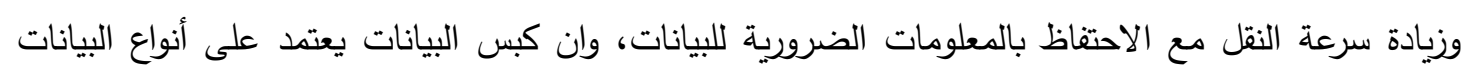

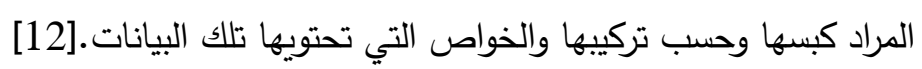

أ- الكبس بدون فقدان (Lossles Compression): في هذا النوع من الكبس من المككن إعادة

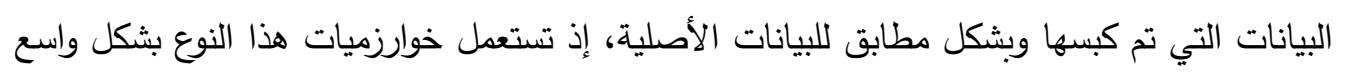

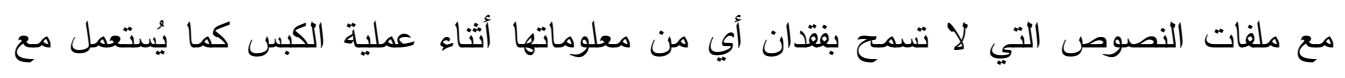

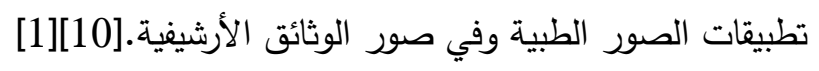
ب- الكبس بفقدان (Lossy Compression): هذا النوع من الكبس يتطلب بعض الخسية الخسارة من البيانات

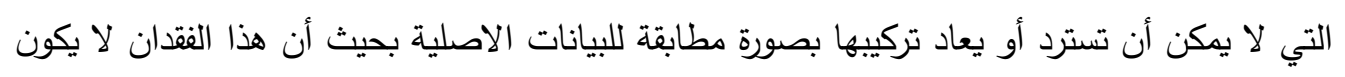

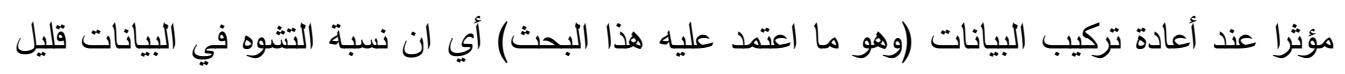

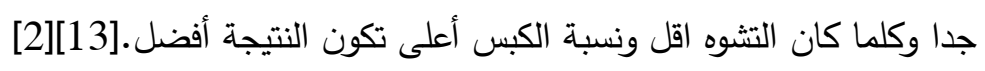

\section{5- بناء الثبكة العصبية الاصطناعية والخوارزمية الجينية:}

\section{Building Artificial Neural Network and Genetic Algorithm:}

الثبكة العصبية الاصطناعية نظام معالجة للمعلومات له مميزات أداء معينة بأسلوب يحاكي الشبكات العصبية الحيوية، إذ أن معمارية الثبكة العصبية النموذجية تتكون من طبقة الإدخال وطبقة الإخراج والطبقة الخفية الوسطية، وتعد شبكة الانتشار العكسي (Back Propagations Network) إحدى أهم الثبكات 
العصبية الاصطناعية متعددة الطبقات[10]، إذ أدت دوراً رئيساً في إعادة بروز الثبكات العصبية الاصطناعية كأداة لحل الكثير من المشاكل على نطاق واسع، وتمكنت هذه الشبكات من حل العديد من المشاكل منها استخلاص الخواص والتصنيف والكبس وغيرها، أما طريقة تدريب هذه الثبكة فتعرف بطريقة التدريب بالانتشار

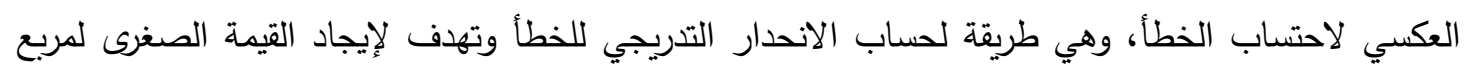
الخطأ الكلي لقيمة الإخراج المحسوب للشبكة[14][15]، تمت عملية التدريب على مرحلتين:

1- مرحلة الانتشار الأمامي: تستقبل فيها كل وحدة إدخال إثارة إدخال ومن ثم تتشر هذه الإشارة إلى كل وحدة من وحدات الطبقة الخفية ثم تحسب كل وحدة من وحدات الطبقة الخفية بدورها قيمة التتشيط لهذه الإشارة وبعدئذ ترسل هذه الوحدات إثارتها إلى كل وحدة من وحدات طبقة الإخراج، ثم تقوم كل طبقة وحنة

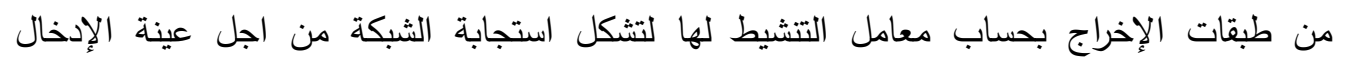

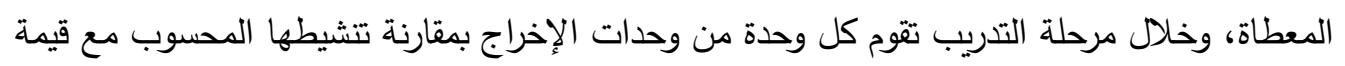
الإخراج الفعلي لتحديد قيمة الخطأ الحاصل لهذه العينة في تلك الوحدة. 2- مرحلة التغذية العكسية: واعتمادا على مقدار الخطأ المحسوب يتم استعمال معامل الخطأ لتوزيع الخطأ على وحدات طبقة الإخراج ويتم نشره بصورة عكسية إلى الوحدات في الطبقات السابقة لاحتساب الخطأ

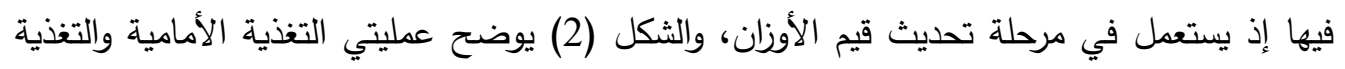
العكسية للشبكة.

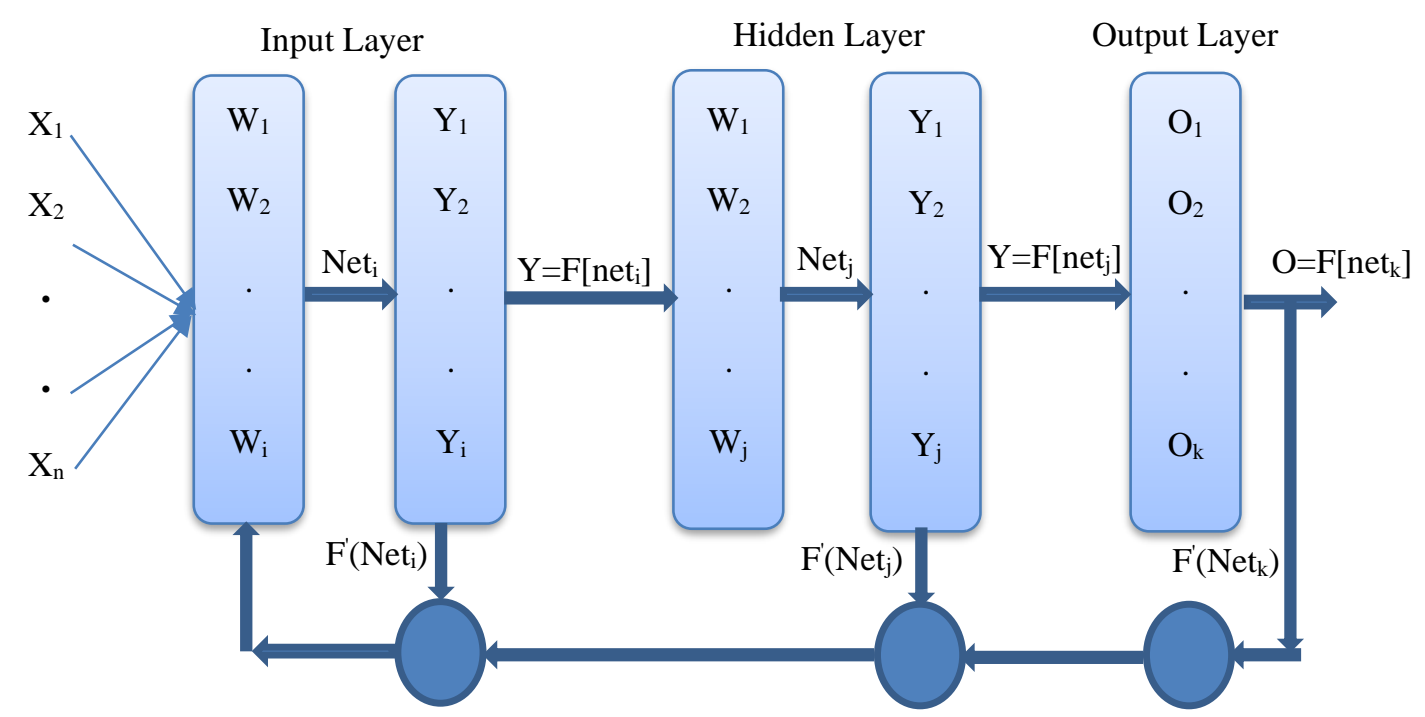

الشكل (2): شبكة الانتشار العكسي (Back Propagations Network)

إذ أن X, Y: يمثلان على التوالي الإدخال والإخراج، و W: تمثل قيمة الوزن، و d: تمثل قيمة

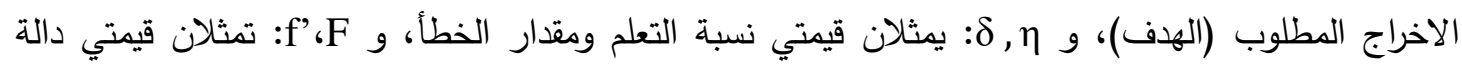
[3][16] التقعيل ومشتقاتها وقد تضمنت خوارزمية التدريب للشبكة الخطوات الآتية:[17][1] 1- تهيئة الأوزان Wقيم Wنيم صغيرة يتم اختيارها بصورة عشوائية ضمن فترة محددة، ثم معالجتها بالخوارزمية 
2- تحديد بيانات التدريب ممثل بالإدخال (الإطار المدخل) والناتج المطلوب وبهذه الصيغة [X

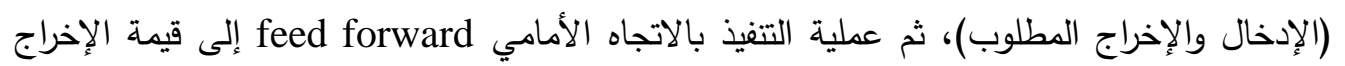

$$
\begin{aligned}
& \text { net }^{\mathrm{L}+1}{ }_{\mathrm{pj}}=\sum_{i=1}^{n} W^{L}{ }_{i \mathrm{j}} \text { out }^{\mathrm{L}}{ }_{\mathrm{i}}+\mathrm{bias}_{\mathrm{j}}^{\mathrm{L}+1}
\end{aligned}
$$

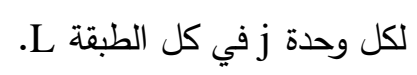$$
\text { out }_{p j}{ }^{\mathrm{L}+1}=\mathrm{F}\left(\text { net }_{\mathrm{pj}}^{\mathrm{L}+1}\right)=1 / 1+\mathrm{e}^{-} \beta^{\text {net } \mathrm{L}+1} \mathrm{pj}
$$

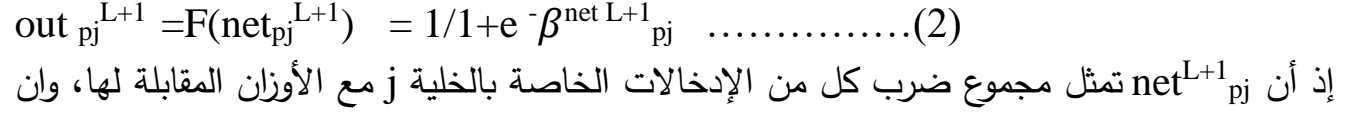

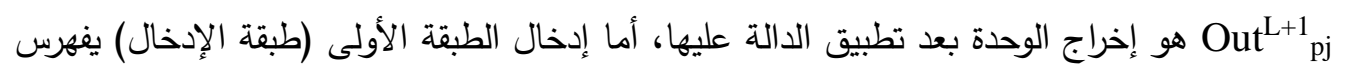

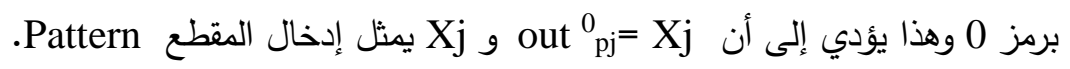
3- حساب الخطأ بين الإخراج الحقيقي للشبكة (out o

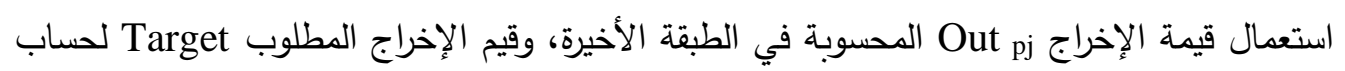
قيمة الـ ل من خلال المعادلة الآتية:

$\boldsymbol{\delta}^{\mathbf{o}} \mathbf{p j}=\left(\mathbf{t}_{\mathbf{p j}}-\mathbf{o u t}^{\mathbf{0}} \mathbf{p j}\right) \mathbf{f}^{\mathbf{e}}\left(\right.$ net $\left.^{0}{ }_{\mathrm{pj}}\right)$

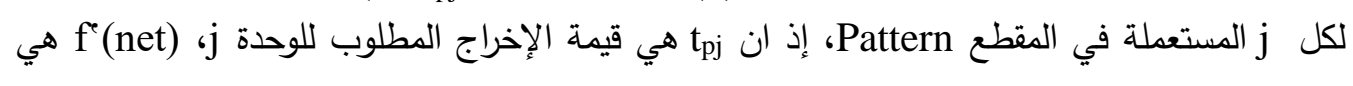
المشتقة لدالة التتشيط (f(net).

4- - حساب قيمة (א) للطبقة الخفية hidden Layer بإسلوب الانتثار العكسي وحسب المعادلة الآتية (إذ

$$
\begin{aligned}
& \text { أن m هي عدد الوحدات في الطبقة L): } \\
& \delta_{\mathrm{pi}}^{\mathrm{L}+1}=\mathrm{f}^{e}\left(\text { net }^{\mathrm{L}+1}{ }_{\mathrm{pi}}\right)\left[\sum_{j=1}^{m L+1} \delta^{\mathrm{L}+2}{ }_{\mathrm{pj} \mathrm{W}}{ }^{\mathrm{L}+1}{ }_{\mathrm{ij}}\right] \\
& W^{\text {new }}{ }_{i j}=w^{\text {old }}{ }_{i j}+\Delta w^{L_{i j}} \\
& \Delta \mathrm{W}^{\mathrm{L}}{ }_{\mathrm{ij}}=\delta \eta_{\mathbf{p j}}{ }^{\mathrm{L}+\mathbf{1}} \mathrm{out}^{\mathrm{L}}{ }_{\mathrm{pi}}
\end{aligned}
$$
3- تنفيذ عملية التغذية الأمامية Forward Activation Function:

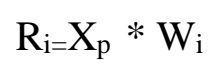

4- إذا لم يتم تجاوز عدد بيانات الاختبار ، اذهب إلى الخطوة الثانية، وإلا اذهب إلى الخطوة الخامسة. 5- نهاية الاختبار ، والشكل (3) يوضح مراحل تتفيذ الخوارزمية. 


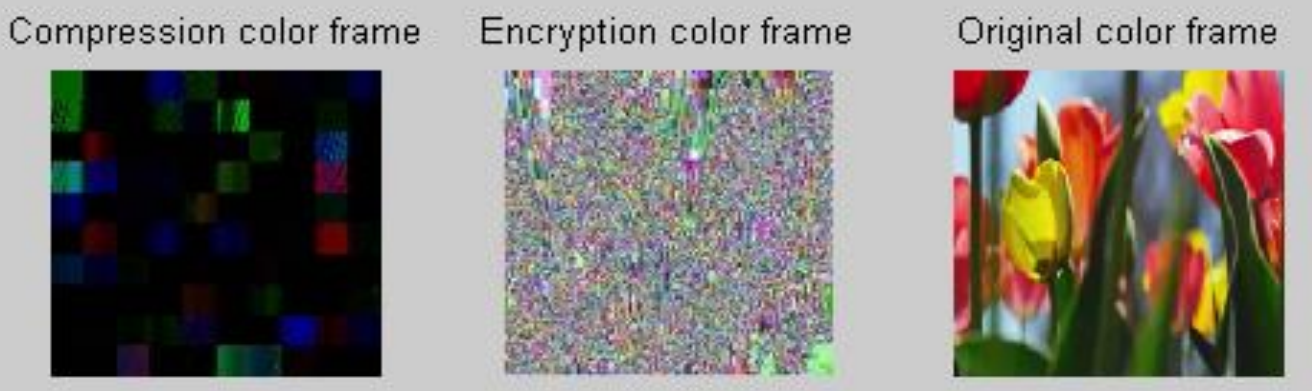

Histogram of compression Histogram of encryption

Histogram of original

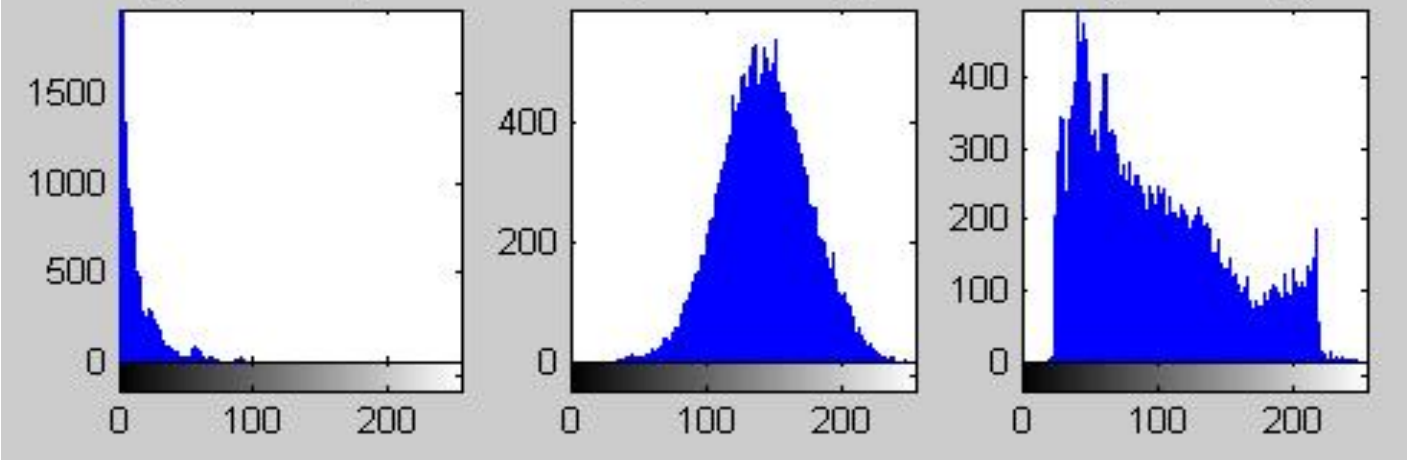

الشكل (3): نتائج التشفير والكبس على الإطار الأول من ملف الفيديو الرقمي

إن عملية حساب الأوزان تمت باستعمال الخوارزمية الجينية، وذلك لأنها تعتبر تقنية بحث تستعمل في

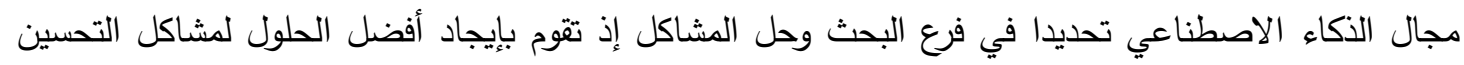
بالاعتماد على العشوائية في البحث.

إذ تتضمن الخوارزمية الجينية عددا من الخطوات الأساسية، هذه الخطوات ثابتة لمختلف المسائل ولكل

التطبيقات ويكون الاختلاف في صيانة كل خطوة من الخطوات وتطبيقها، إذ أن خطوات المسألة مترابطة بعضها

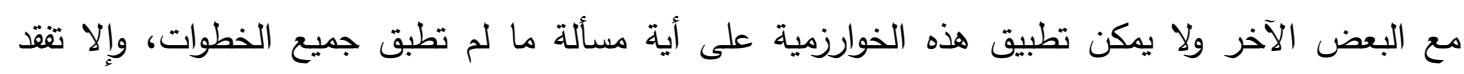
الخوارزمية الجينية قيمتها وفائدتها في إيجاد الحل أو تحسينه، والمخطط (3) يوضنح آلية آلية عمل الخوارزمية 


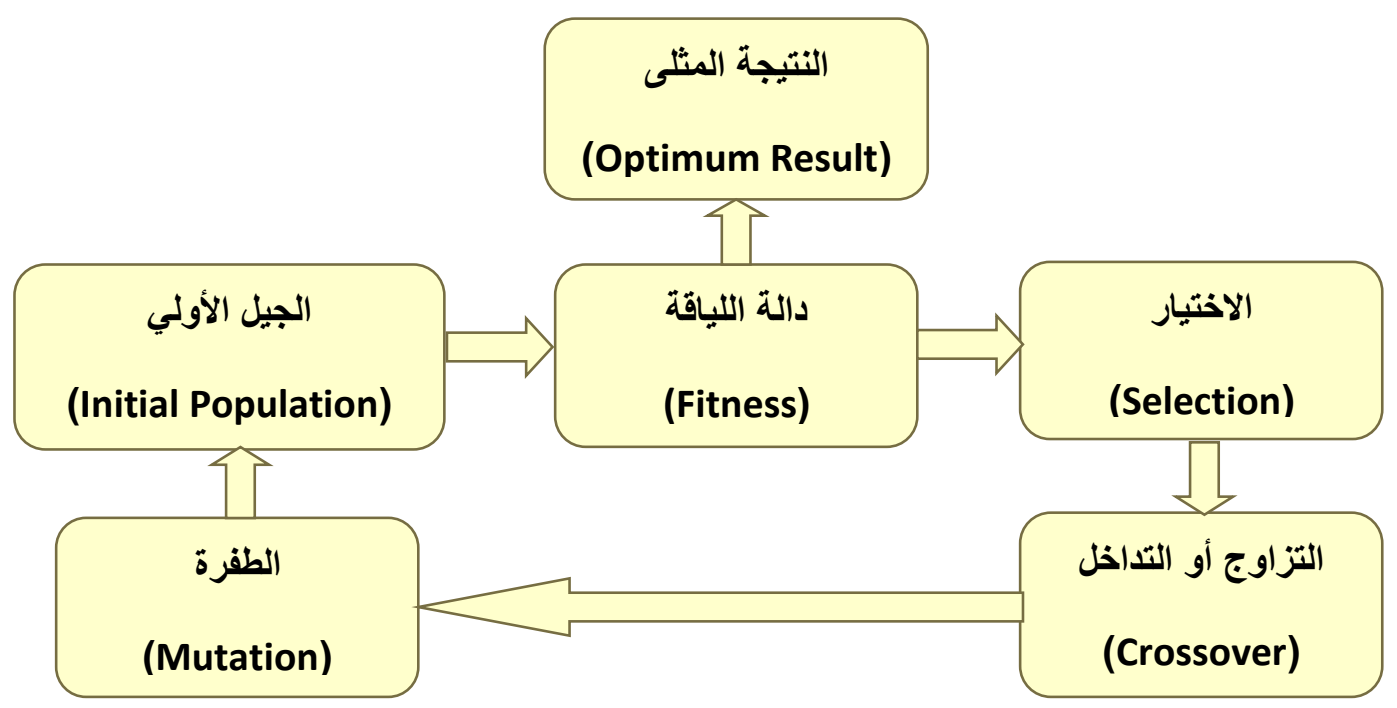

المخطط (3): خطوات عمل الخوارزمية الجينية

أما خطوات عمل الخوارزمية الجينية المقترحة: 1- ت تكوين جيل عشوائي من N من الكروموسوم وM من الجينات. 2- حساب دالة اللياقة (fitness function) لكل كروموسوم في الجيل الحالي. 3- توليد جيل جديد (new population) بتكرار الخطوات الآتية حتى يكتمل الجيل، إذ يجب أن يحتوي الكروموسوم على معلومات عن الحل الذي سوف يقدمه وقد تم استعمال طريقة الثريط الثنائي إذ يمثل كل إنل كروموسوم بواسطة سلسلة ثنائية تحوي عدد من البتات يمكن لكل بت في السلسلة أن يمثل بعض المبل الميزات

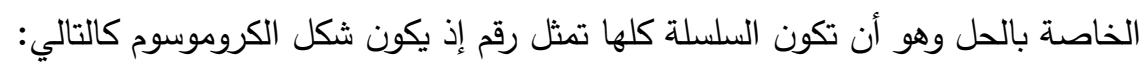

Chromosome1: 1101100100110110

Chromosome2: 1100111000011110

يتم اختيار (selection) كروموسومان ليمثلا الوالدين من الجيل الحالي بالاعتماد على دالة اللياقة

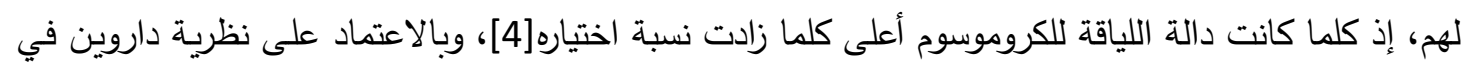
التطور فإن الأفضل في الجماعة هو الذي يشكل الجيل الجديد، ويمكن وصف هذه العملية من خلال الخطوات

أ- الجمع: حساب مجموع لياقة كل الكروموسومات في الجماعة ويرمز له بالرمز (S). ب- الاختيار : إختيار رقم عشوائي من المجال (0,S) ويرمز لله بالرمز (r ). 
ت- الحلقة: جمع كل اللياقات في الجماعة من الصفر وحتى S الى أن يصبح S أكبر من r يتم

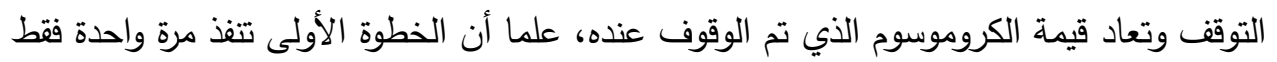
من أجل كل جماعة.

4- يتم إجراء عبور أو تزاوج (crossover) بين الكروموسومين عند نقطسة التزاوج إذا كـان لديهما احتماليـة تزاوج، ويتم التزاوج بانقسام كل كروموسوم عند نقطة التزاوج، ثم يوصل كل جزء من احد الكروموسومين مـع

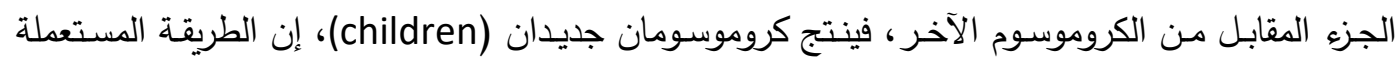

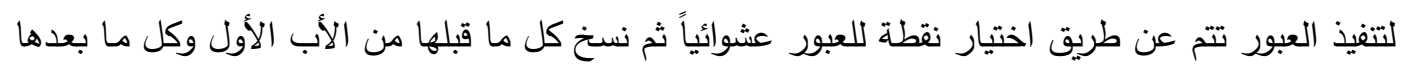

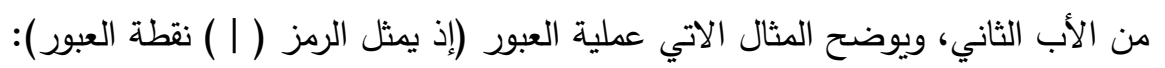

Chromosome 1: 11011 |00100110110

Chromosome 2: $11001 \mid 11000011110$

Offspring 1: $\quad 11011 \mid 11000011110$

Offspring 2: $11001 \mid 00100110110$

إذا لم يكن هناك عبور فإن الجيل الجديد الناتج سوف يكون نسخة مطابقة للأبوين أما إذا تم العبور

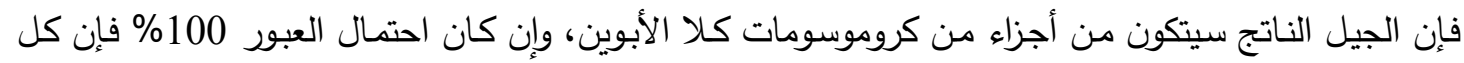

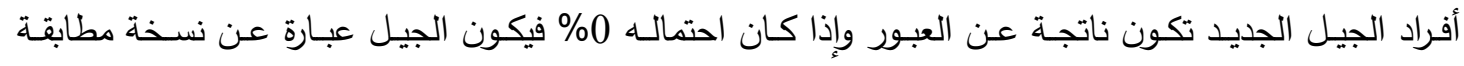

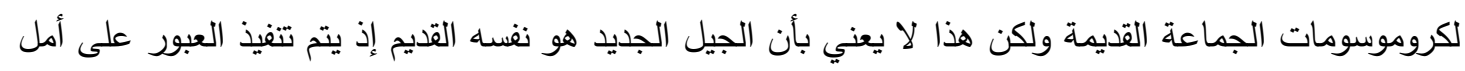

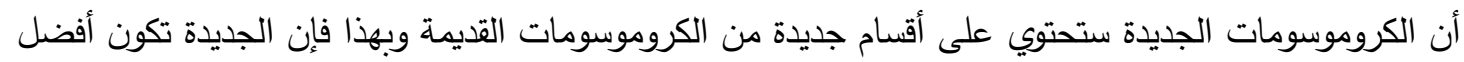

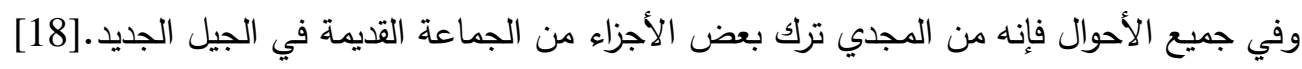

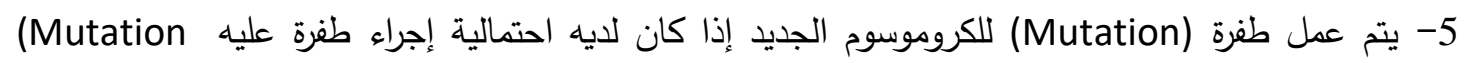

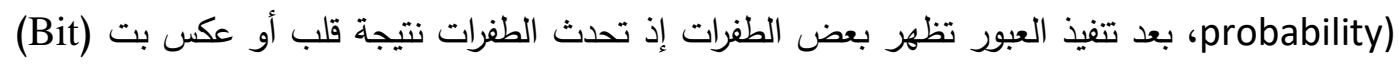
وان الغاية من الطفرة هي منع الوقوع في مشكلة كون كل الحلول في الجماعة هي حل مثالي للمسألة المراد حلها[17]، وإن تثفير الطفرات بالإضافة إلى العبور تعتمد بشكل أساسي على تشفير الكروموسومات إذ فئ

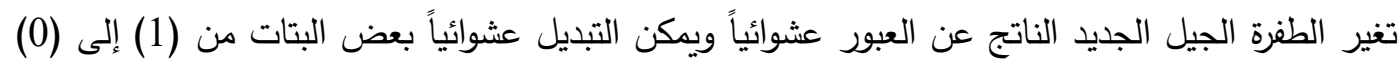

Original offspring 1: 1101111000011110

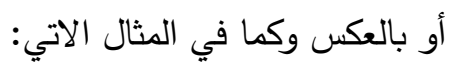

Original offspring 2: 1100100100110110

Mutated offspring 1: 1100111000011110

Mutated offspring 2: 1100101100110100

إذا لم تحدث طفرات فإن الجيل الجديد يتشكل مباشرة بعد العبور (ينسخ مباشرة) بدون أي تغييرات أما

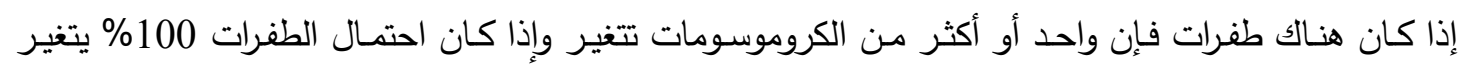

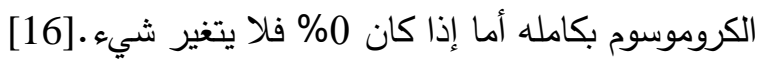
بشكل عام تمنع الطفرات الخوارزميات الجينية من الوقوع في نهاية محلية وينبغي أن لا تتم الطفرات بكثرة لأن الخوارزمية الجينية عندها ستتحول إلى بحث عشوائي(Random search). 6- عملية الاستبدال (accepting) وهي عملية إضافة الكروموسوم الناتج إلى الجيل الجديد.[13]

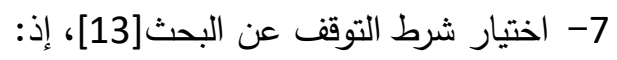


أ- تحقق شرط التوقف: يتم التوقف للخوارزمية الجينية، وإعادة الحل الجيد من آخر جيل متكون. ب- عدم تحقق شرط التوقف: الرجوع إلى الخطوة (2)، إذ أن كل تكرار لهذه العملية يسمى بالجيل الجديد (Generation) )، وبعد نهاية التنفيذ يتم تقديم تقرير عن الحقائق التي تم التوصل إليها.

\section{6-فاك الكبس والتشفير Decompression and Decryption:}

بعد أن تمت عملية التشفير والكبس، يتم فلك تشفير وكبس ملفات الفيديو وذلك من خلال تتفيذ عمليات

معاكسة لعملية التشفير والكبس، وكما في المخطط الانسيابي (4).

إذ يتم العمل على إدخال ملف الفيديو المشفر والمكبوس ثم تحليل اطر الفيديو إلى الثرائح اللونية

الثلاث (الأحمر والأخضر والأزرق)، ثم استرجاع قيم الأوزان التي تم الاحتفاظ بها والناتجة من الخوارزمية

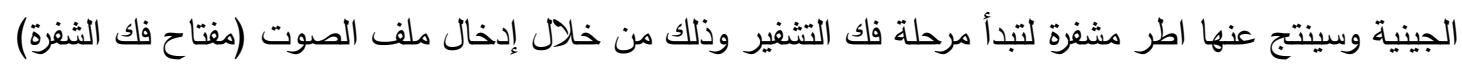
ثم عمل XOR مع الأطر المشفرة لينتج بعد ذلك ملف الفيديو غير المشفر وغير المكبوس، والثكل (4) يوضح

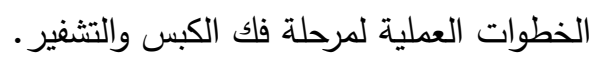

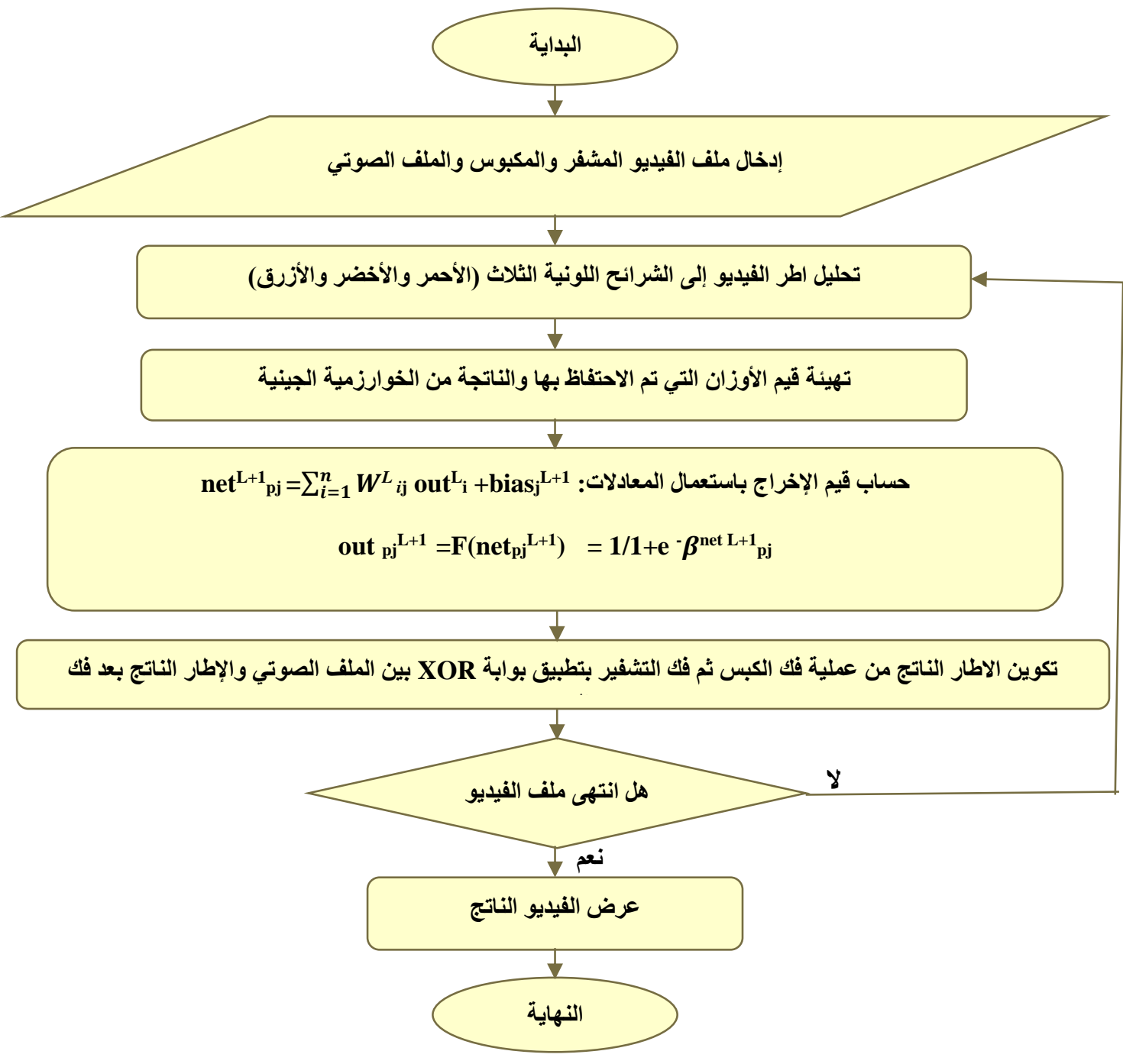

المخطط الانسيابي (4): خطوات فلك الكبس والتشفير 


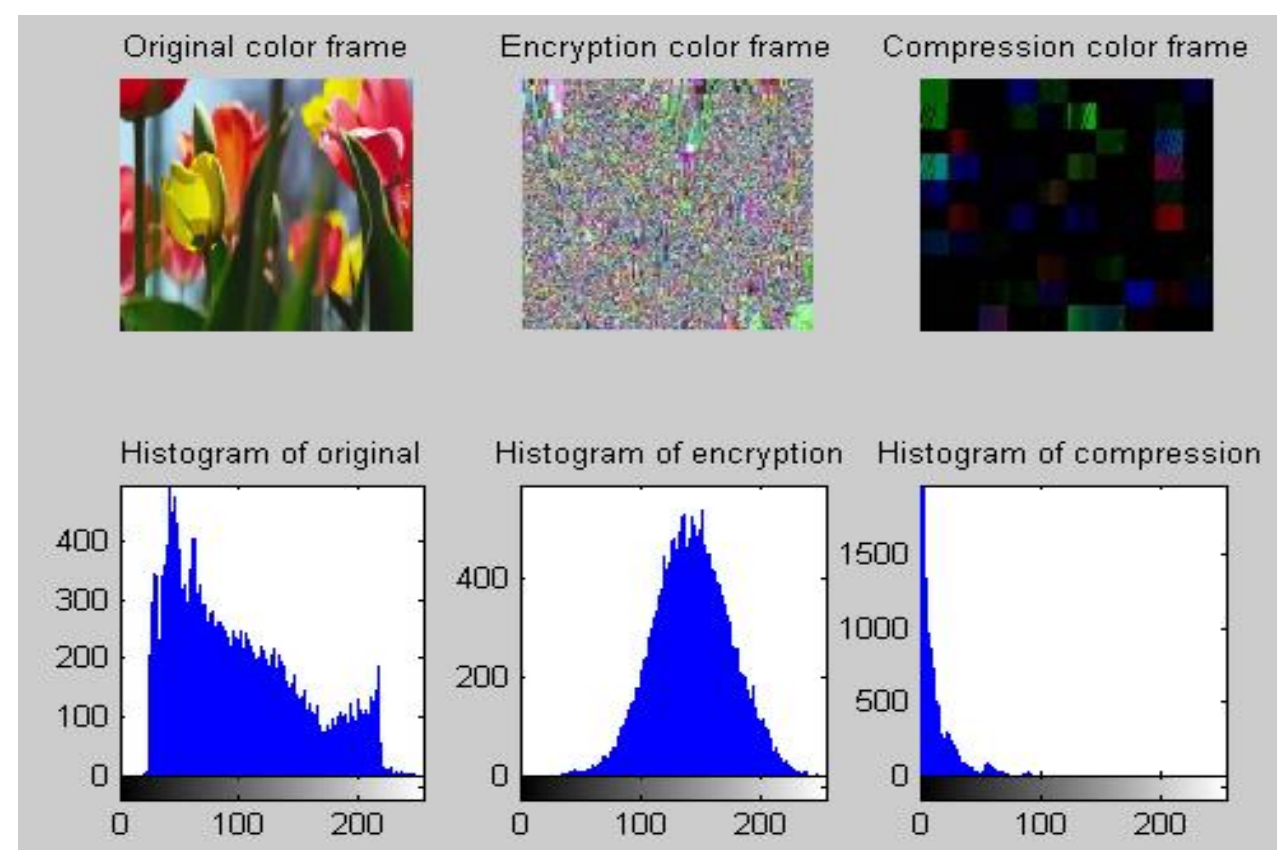

الشكل (4): نتائج فلك الكبس وفك التشفير على الإطار الأول من ملف الفيديو

Measures Of Accuracy

بعد أن تم تطبيق جميع الخوارزميات والحصول على نتائجها، تم حساب نسبة الكبس وتقييم النتائج

بوساطة اختبارات الدقة وذلك عبر استعمال طرائق رياضية، وتوجد عدة أنواع من الاختبارات الإحصائية أهمها:

1- إيجاد اقل قيمة لمربع الخطأ (Minimum Squared Error) بين إثارة الإدخال والإخراج، إذ تمثل M و و

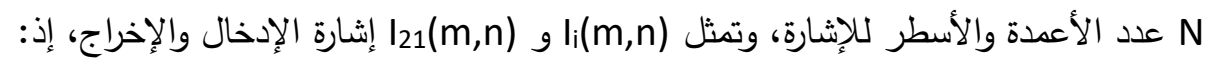

MSE $=\left(\sum_{M N}\left[I_{i}(m, n)-I_{21}(m, n)\right]^{2}\right) /(M * N)$

2- قياس نسبة الضوضاء (Peak Signal-to-noise ratio): إذ تمثل R ميم البيانات إذا كانت :Unsigned integer أو Floating Point

$P S N R=10 \log _{10}\left[R^{2} / M S E\right]$

3- معامل الارتباط (Correlation): هو مقياس لقوّة العلاقةِ بين المتغيرات العشوائية، إذ:

$\operatorname{Corr}=\frac{\sum\left(I-\mu_{I}\right)\left(O-\mu_{O}\right)}{\sqrt{\sum\left(I-\mu_{I}\right)^{2} \sum\left(O-\mu_{O}\right)^{2}}}$

ومعامل الارتباط يمكن أن يأخذ القيم بين (-1 و+1)، إذ أن الإثارة (+ أو -) لمعامل الارتباط تمثل

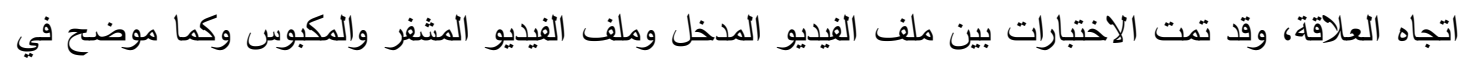
الثكل (5)، وأيضا تمت الاختبارات بين ملف الفيديو المدخل وملف الفيديو بعد الاسترجاع وكما في الثكل (6). 
من خلال متابعة الثكل(5) والثكل(6) تبين بان معامل الارتباط كان قريبا من الصفر في الثكل (5)

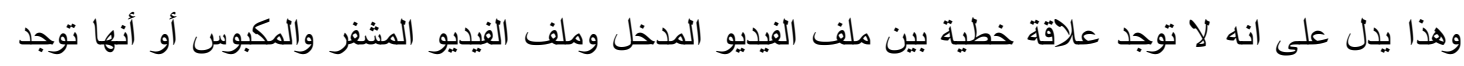
علاقة خطية ضعيفة جدا، وأما في الثكل (6) فان معامل الارتباط (بين ملف الفيديو المدخل وملف الفيديو بعد فلك التثفير وفك الكبس) قربب من الواحد وهذا يدل على وجود علاقة خطية قوية جدا وقد تكون مثالية.
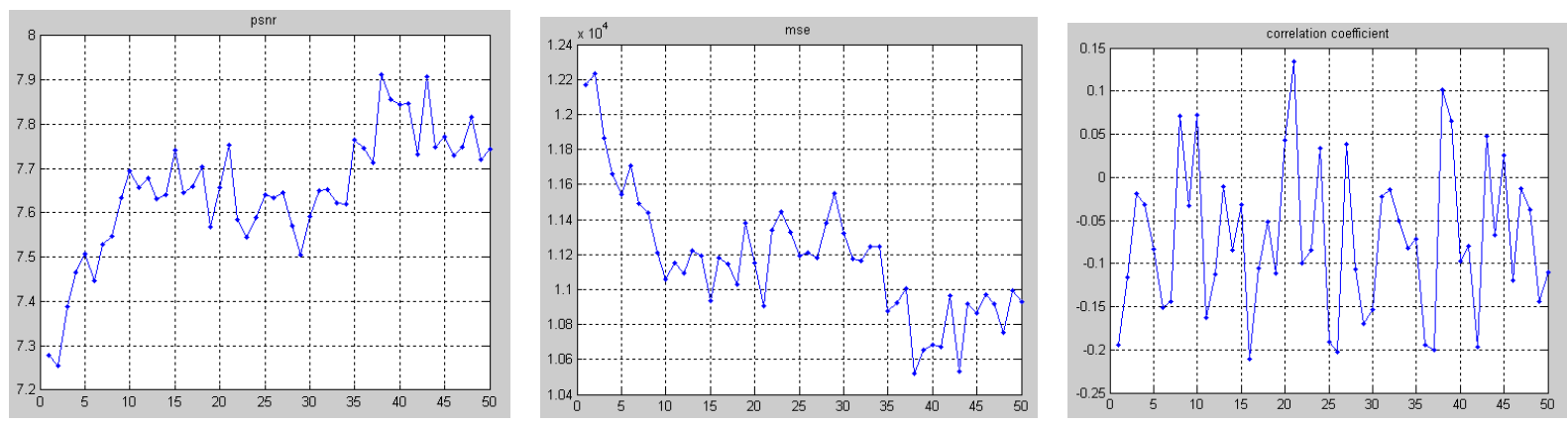

الثكل (5): تطبيق مقاييس الدقة على ملف الفيديو قبل وبعد التشفير والكبس
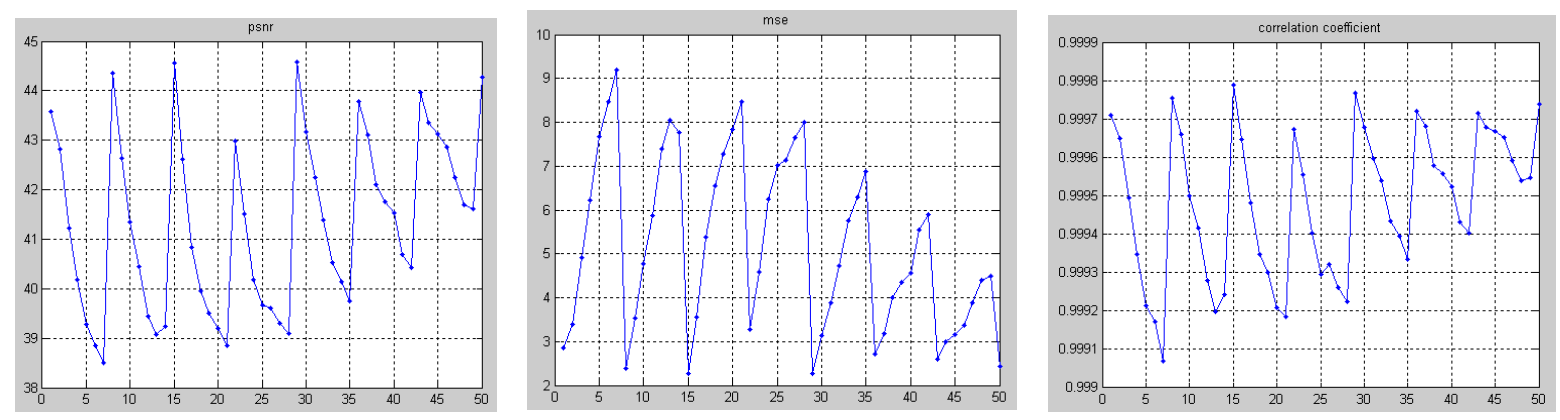

الثكل (6): تطبيق مقاييس الدقة على ملف الثيديو المدخل وملف الفيديو بعد الاسترجاع

وأما قيمة مربع الخطأ (MSE) ففي الثكل (5) كانت قيمها كبيرة جدا وهذا يدل على انه لا يوجد ارتباط بين ملفي الفيديو، وفي الثكل (6) فان قيم مربع الخطأ صغيرة جدا وهذا يدل على انه يوجد ارتباط بين ملفي الثيديو، والجدول (1) يوضح نتائج التتفيذ على 10 عينات متتوعة من ملفات الفيديو الرقمي المدخلة.

\begin{tabular}{|c|c|c|c|c|c|c|c|c|}
\hline \multirow{2}{*}{$\begin{array}{c}\text { Video } \\
\text { NO. }\end{array}$} & \multicolumn{2}{|c|}{$\begin{array}{c}\text { Encryption } \\
\text { Operation }\end{array}$} & \multicolumn{2}{c|}{$\begin{array}{c}\text { Compression } \\
\text { Operation }\end{array}$} & $\begin{array}{c}\text { Compression } \\
\text { Ratio }\end{array}$ & $\begin{array}{c}\text { NO. of } \\
\text { Frames }\end{array}$ & \multicolumn{2}{c|}{$\begin{array}{c}\text { Dimension of } \\
\text { Frames }\end{array}$} \\
\cline { 2 - 4 } \cline { 8 - 10 } & MSE & PSNR & MSE & PSNR & & & Height & width \\
\hline Video1 & $1.3962 \mathrm{e}+04$ & 6.6814 & $4.1203 \mathrm{e}+03$ & 11.9815 & 1.89804 & 92 & 300 & 300 \\
\hline Video2 & $1.1377 \mathrm{e}+04$ & 7.5707 & $2.8919 \mathrm{e}+03$ & 13.5190 & 2.12476 & 20 & 300 & 300 \\
\hline Video3 & $7.9527 \mathrm{e}+03$ & 9.1256 & $3.5802 \mathrm{e}+03$ & 12.5918 & 2.19106 & 102 & 300 & 300 \\
\hline Video4 & $9.3162 \mathrm{e}+03$ & 8.4384 & $4.1367 \mathrm{e}+03$ & 11.9643 & 2.08143 & 120 & 300 & 300 \\
\hline Video5 & $1.0306 \mathrm{e}+04$ & 7.9998 & $4.0038 \mathrm{e}+03$ & 12.1061 & 2.08516 & 143 & 300 & 300 \\
\hline Video6 & $8.5195 \mathrm{e}+03$ & 8.8267 & $3.5058 \mathrm{e}+03$ & 12.6829 & 2.24880 & 88 & 300 & 300 \\
\hline Video7 & $1.2760 \mathrm{e}+04$ & 7.0724 & $3.8236 \mathrm{e}+03$ & 12.3061 & 2.06485 & 114 & 300 & 300 \\
\hline Video8 & $9.2516 \mathrm{e}+03$ & 8.4686 & $3.3574 \mathrm{e}+03$ & 12.8708 & 2.25410 & 51 & 300 & 300 \\
\hline Video9 & $9.8087 \mathrm{e}+03$ & 8.2147 & $4.0155 \mathrm{e}+03$ & 12.0934 & 2.05042 & 45 & 300 & 300 \\
\hline Video10 & $1.0723 \mathrm{e}+04$ & 7.8275 & $3.8581 \mathrm{e}+03$ & 12.2671 & 2.11644 & 58 & 300 & 300 \\
\hline
\end{tabular}

الجدول (1): نتائج التتفيذ على 10 عينات متتوعة من الفيديو الرقمي 
بعد تطبيق خوارزميات التشفير والكبس وتطبيق خوارزميات فك التشفير وفك الكبس وبعد الحصول على النتائج وقيم مقاييس الدقة تبين بان عملية تشفير وكبس ملفات الفيديو الرقمي أعطت نتائج تتاسبت مع فئي

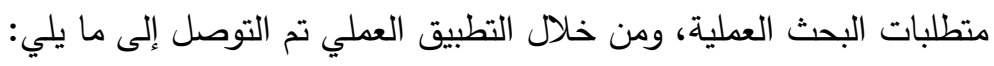

1- إن التشفير باستعمال بوابة XOR بين الإطار المدخل والمقطع الصوتي (مفتاح التشفير) تطابقت مع

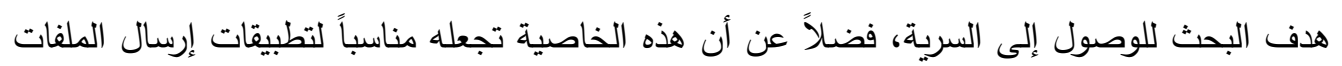

$$
\text { الفيديوية عبر وسائط النقل (كالانترنت). }
$$

2- إن تطبيق الشبكة العصبية الاصطناعية (شبكة انتشار الخطأ خلفيا) مع الخوارزمية الجينية لكبس ملفات الفيديو الرقمي أعطت نتائج متوافقة مع متطلبات البحث، وذلك عبر نتائج زمن التتفيذ والنسبة المئوية للكبس ومقاييس الدقة، فمن خلال ملاحظة النتائج في الجدول رقم (1) تبين أن نسبة الكبس الكبات

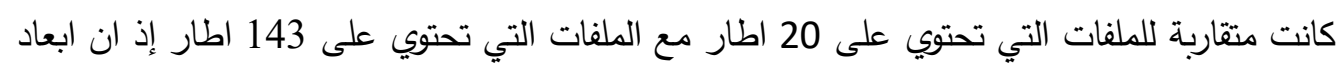
الاطر هي 300*300 وان حجم الفيديو الناتج اصبح اقل من نصف حجم الفيديو الاصلي، من جهة الفئ اخرى فان الزيادة في الارقام الناتجة لقيم الـMSE تدل على كفان كفاءة التشفير والكبس تقابلها الارقام

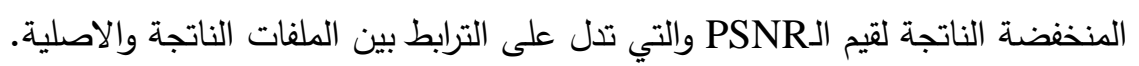

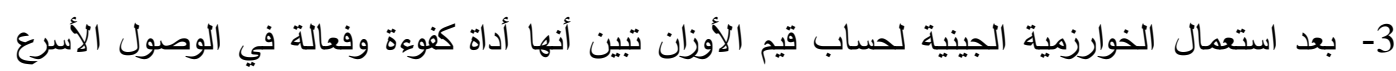
إلى النتائج المطلوبة لما لها من ميزات تساعد في تسهيل معالجة البيانات الفيديوية وتحضيرها لعملية الكبس من خلال الوصول الأمثل للأوزان المعتمدة. 4- إن عملية العنقدة وتقسيم الأطر إلى مجموعة من العناقيد (النقاط الضوئية المتجانسة) أدت إلى إعطاء

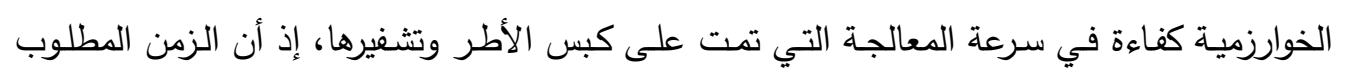
لكبس إطار واحد من أطر الفيديو الرقمي هو 10 ثانية تقريبا.

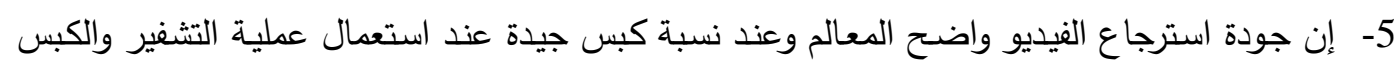

$$
\text { على الفيديو المستعمل. }
$$

\section{References المصادر}

1- Asbun E., Thesis, Submitted to the Faculty of Purdue University, In Partial Ful_llment of the Requirements for the Degree of Doctor of Philosophy, 2000.

2- Han B., Wu D., Zhang H., Thesis, Department of Electrical and Computer Engineering, University of Florida Gainesville, FL 32611, Cisco Systems, Santa Clara, Provider: citeseer, 2013.

3- Kin C. Y. S., Coker B., Thesis, Electrical Engineering cedyue@stanford.edu, Computer Science bcoker@stanford.edu, 2017.

4- Choudhary R., Abrol P., Thesis, International Journal of Advanced Research in Computer Engineering \& Technology (IJARCET) Volume 6, Issue 6, June 2, ISSN: $2278-1323,2017$.

5- Zhang X., Wu X., Thesis, Fellow, IEEE, MANUSCRIPT SUBMITTED TO IEEE TRANSACTIONS ON IMAGE PROCESSING, arXiv:1801.07987v2 [cs.CV] 19 Mar, 2018. 
6- Gloe T., Fischer A., Kirchner M., Thesis, Journal: Digital Investigation ISSN: 17422876, Volume: 11 Issue: -Supplement_1 Pages: S68-S76 Provider: Elsevier DOI: 10.1016/j.diin.2014.03.009, 2014.

7- Xu D., Wang R., Shi Y. Q., Thesis, Journal: Information Forensics and Security, IEEE Transactions on ISSN: 15566013, Volume: 9 Issue: 4 Pages: 596-606 Provider: IEEE Publisher: IEEEDOI: 10.1109/TIFS.2014.2302899, 2014.

8- Srikanth P., Mehta A., Yadav N., Singh S., Singhal S., Thesis, IJCSN International Journal of Computer Science and Network, Volume 6, Issue 3, June ISSN (Online): 2277-5420, 2017.

9- Um H. Y., Thesis, Submitted to the Faculty of Purdue University, Purdue University West Lafayette, Indiana, 2006.

10- Anand A. and Suganthi L., Thesis, Energies, 11, 728; doi:10.3390/en11040728, 2018.

11-Xie L., Yuille A., Thesis, Center for Imaging Science, The Johns Hopkins University, Baltimore, MD, USA, arXiv:1703.01513v1 [cs.CV] 4 Mar, 2017.

12- Li M., Gu S., Zhang D., Thesis, arXiv:1801.04662v1 [cs.CV] 15 Jan, 2018.

13- Pradeep S. A., Manavalan R., Thesis, International Journal of Engineering Research Volume No.2, Issue No. 6, pp : 386-392, 2013.

14- Beale M. H., Hagan M.T., Demuth H. B., "Neural Network Toolbox", User's Guide, by The MathWorks, Inc., www.mathworks.com, 2014.

15- Suganuma M., Shirakawa S., Nagao T., Thesis, arXiv:1704.00764v2 [cs.NE] 11 Aug, 2017.

16- Mohammadi M., Mehrolhassani M. A., Thesis, Under review as a conference paper at ICLR, 2017.

17- Kaviani M., MirRokni S. M., Thesis, IJCSNS International Journal of Computer Science and Network Security, VOL.17 No.6, June, 2017.

18- Wang F., Junlan L., Liu S., Zhao X., Zhang D., Tian Y., Thesis, Journal: Mechatronics, IEEE / ASME Transactions on ISSN: 10834435, Volume: 19 Issue: 3 Pages: 916-923 Provider: IEEE Publisher: IEEE DOI: 10.1109/TMECH.2013.2260555, 2014. 